\title{
Análise Documental sobre as Normativas do Trabalho no Núcleo Ampliado de Saúde da Família
}

\author{
Meyrielle Belotti ${ }^{1}$ \\ ${ }^{1}$ Universidade Federal do Espírito Santo, ES, Brasil. \\ Luziane Zacché Avellar ${ }^{3}$ \\ ${ }^{3}$ Universidade Federal do Espírito Santo, ES, Brasil.
}

\begin{abstract}
Resumo: O artigo tem como objetivo analisar os principais documentos do Ministério da Saúde, publicados entre os anos de 2010 a 2014, que orientam o trabalho do Núcleo Ampliado de Saúde da Família (NASF) e discorrem sobre suas diretrizes. Para tanto, utilizou-se da técnica de análise documental. Foram analisados os seguintes documentos: Políticas Nacional da Atenção Básica, promulgada em 2012, Cadernos da Atenção Básica no 27 e no 39. A análise dos dados foi realizada com base na análise de conteúdo. Os resultados foram categorizados da seguinte forma: as diretrizes do NASF como guia do processo de trabalho; metodologia e ferramentas de trabalho; e a normativa para organização do trabalho. Verificou-se que o trabalho do NASF permite a realização de diversas ações a partir das necessidades das equipes de Saúde da Família (eSF) e do território. Além disto, observou-se que as normativas do NASF propõem uma lógica de organização do trabalho em saúde, pautada em uma atuação conjunta, construída por meio de interação e pactuação estabelecidas entre as equipes NASF, as eSF, os gestores, a comunidade e os demais equipamentos sociais existentes do território.
\end{abstract}

Palavras-chave: Atenção Primária à Saúde, Núcleo Ampliado de Saúde da Família, Trabalho em Saúde, Pesquisa Documental.

\section{Documentary Analysis on Labor Standards at the Family Health Expanded Support Center}

\begin{abstract}
The article aims to analyze the main documents of the Ministry of Health, published between 2010 to 2014, which guide the work of the Family Health Expanded Support Center (NASF) and discuss its guidelines. For this purpose, the documentary analysis technique was used. The following documents were analyzed: National Policies for Basic Care, promulgated in 2012 and Notebooks for Basic Care $n^{\circ} 27$ and no 39. Data analysis was performed based on content analysis. The results were categorized as follows: the NASF guidelines as a guide to the work process; methodology and tools of work and the norms for work organization. It was verified that the work of the NASF allows the accomplishment of several actions from the needs of the Teams of Family Health (eSF) and of the territory. In addition, it was observed that the regulations of the NASF propose a logic of organization of work in health, based on a joint action, built through the interaction and agreement established between NASF, eSF, managers, community and other existing social equipment teams of the territory.
\end{abstract}

Keywords: Primary Health Care, Family Health Expanded Support Center, Health Work, Documentary Research. 


\title{
Análisis Documental sobre las Normativas del Trabajo en el Núcleo Ampliado de Salud de la Familia
}

\begin{abstract}
Resumen: El artículo tiene como objetivo analizar los principales documentos del Ministerio de Salud, publicados entre los años 2010 a 2014, que orientan el trabajo del Núcleo Ampliado de Salud de la Familia (NASF) y discurren sobre sus directrices. Para ello, se utilizó la técnica de análisis documental. Se analizaron los siguientes documentos: Políticas Nacional de la Atención Básica, promulgada en 2012, Cuadernos de la Atención Básica n 27 y n 39 . El análisis de los datos fue realizado con base en el análisis de contenido. Los resultados se clasificaron de la siguiente manera: las directrices del NASF como guía del proceso de trabajo; metodología y herramientas de trabajo y la normativa para la organización del trabajo. Se verificó que el trabajo del NASF permite la realización de diversas acciones a partir de las necesidades de los equipos de Salud de la Familia (ESF) y del territorio. Además, se observó que las normativas del NASF proponen una lógica de organización del trabajo en salud, pautada en una actuación conjunta, construida por medio de interacción y pactos establecidos entre los equipos NASF, las eS, los gestores, la comunidad y demás equipos sociales existentes del territorio.
\end{abstract}

Palabras clave: Atención Primaria a la Salud, Núcleo Ampliado de Salud de la Familia, Trabajo en Salud, Investigación Documental.

\section{Introdução}

O Ministério da Saúde visando superar a lógica dos processos de trabalho fragmentado e individual, em direção à gestão integrada e a corresponsabilização do cuidado, instituiu o Núcleo de Apoio à Saúde da Família (NASF), por meio da promulgação da Portaria $n^{\circ} 154 / 2008$. O NASF é constituído por categorias profissionais de diferentes áreas de conhecimento ${ }^{1}$, que atuam no formato do trabalho em equipe, apoiando às equipes de Saúde da Família (eSF), com o objetivo de ampliar a resolutividade das ações na Atenção Básica (AB).

Em síntese, o trabalho do NASF acontece a partir da integração com a eSF, que juntos são responsáveis pelo cuidado em um determinado território. O NASF é composto por profissionais de núcleos de conhecimento diferentes dos trabalhadores que compõem as eSF (Matuda, Pinto, Martins, \& Frazão, 2015). Neste contexto, o NASF caracteriza-se como um dispositivo que incorpora novos saberes ao trabalho em saúde na Atenção Básica, por meio do desenvolvimento de práticas conjuntas com as eSF, elaboradas a partir da ativação de espaços para o compartilhamento de conhecimentos, com vistas a ampliação do cuidado.
Verifica-se que o NASF representou um passo importante para o fortalecimento da eSF e especialmente para o desenvolvimento e aprimoramento de um modelo de trabalho em equipe multiprofissional.

Ressalta-se que a implantação dos NASF no país encontra-se em processo de consolidação. Os documentos ministeriais que normatizam os NASF são recentes e caminham rumo a sua operacionalização. Além disto, devido ao seu ineditismo e pouco tempo de implantação, os estudos que abordam esta temática ainda são escassos, principalmente aqueles que enfocam a análise das políticas que o regulamentam (Anjos, et al., 2013; Bonladi, \& Ribeiro, 2014; Gonçalves, Lancman, Sznelwar, Cordone, \& Barros, 2015).

De acordo com Paim (2003), a compreensão de uma política de saúde, assim como a definição de diretrizes, programas e princípios são fundamentais na implementação de qualquer ação em saúde. Nesse sentido, este artigo tem como objetivo analisar os principais documentos do Ministério da Saúde, que orientam o trabalho do NASF e discorrem sobre suas diretrizes. Entende-se que a compreensão destes documentos se constituí como um dos elementos essenciais para a concretização da proposta do NASF.

${ }^{1}$ Médico Acupunturista, Assistente Social, Profissional da Educação Física, Farmacêutico, Fisioterapeuta, Fonoaudiólogo, Médico Ginecologista, Médico Homeopata, Nutricionista, Médico Pediatra, Psicólogo, Médico Psiquiatra e Terapeuta Ocupacional. 


\section{Metodologia}

Trata-se de uma pesquisa documental, que de acordo com Oliveira (2007) se assemelha à pesquisa bibliográfica, mas requer do pesquisador "uma análise mais cuidadosa, visto que os documentos não passaram antes por nenhum tratamento científico" (p. 70).

Para tanto, nesta pesquisa, foram analisados os principais documentos do Ministério da Saúde, publicados entre os anos de 2010 a 2014, que orientam o trabalho do NASF e discorrem sobre suas diretrizes, a saber: 1) o Caderno da Atenção Básica, no 27, intitulado "Diretrizes do NASF: Núcleo de Apoio a Saúde da Família"; 2) a Políticas Nacional da Atenção Básica, que inclui os NASF neste nível de complexidade; e 3) o Caderno de Atenção Básica, no 39, intitulado “Núcleo de Apoio à Saúde da Família - V. 1: Ferramentas para a gestão e para o trabalho cotidiano". O Quadro apresenta a caracterização dos documentos analisados.

A análise dos dados foi realizada com base em alguns pressupostos da análise de conteúdo, propostos por Bardin (2009). Assim, os dados foram sistematizados da seguinte forma: 1) pré-leitura dos docu- mentos, visando ter uma visão global destes; 2) leitura seletiva, em que se buscou identificar as informações pertinentes ao objetivo do estudo, sendo destacadas as frases acerca das normativas para organização do trabalho do NASF e suas diretrizes; 3) categorização dos trechos identificados, que foram agrupados e classificados por semelhança, a partir da leitura seletiva e, por fim, 4) análise descritiva e reflexiva dos dados.

\section{Resultados e discussão}

Conforme processo de análise detalhado acima, os dados analisados delinearam três categorias: (1) As diretrizes do NASF como guia do processo de trabalho; (2) Metodologia e Ferramentas de trabalho e (3) A organização do trabalho. Nesta última categoria, devido à diversidade dos temas encontrados durante a análise, optou-se por apresentar os dados em subcategorias, a saber: o acesso aos profissionais do NASF; a organização da agenda de trabalho e; o planejamento das ações. A discussão dos resultados foi realizada concomitante à apresentação dos dados.

\section{Quadro}

Caracterização dos documentos analisados.

\begin{tabular}{|c|c|c|}
\hline Documento & Fonte/ano & Proposta \\
\hline $\begin{array}{c}\text { Caderno da Atenção } \\
\text { Básica, no 27, Diretrizes } \\
\text { do NASF: Núcleo de } \\
\text { Apoio a Saúde da Família }\end{array}$ & $\begin{array}{l}\text { Ministério da Saúde/ } \\
\text { Secretaria de Atenção à } \\
\text { Saúde/Departamento de } \\
\text { Atenção Básica, } 2010\end{array}$ & $\begin{array}{c}\text { Complementar as orientações definidas por meio da } \\
\text { Portaria no } 154 / 2008 \text {, visando traçar diretriz do NASF, } \\
\text { mais claras para gestores e trabalhadores e fortalecer } \\
\text { a AB no país, por meio do aumento do conhecimento } \\
\text { sobre a temática do NASF, nas equipes que atuam neste } \\
\text { nível de complexidade. }\end{array}$ \\
\hline $\begin{array}{l}\text { Políticas Nacional da } \\
\text { Atenção Básica }\end{array}$ & $\begin{array}{l}\text { Ministério da Saúde/ } \\
\text { Secretaria de Atenção à } \\
\text { Saúde/Departamento de } \\
\text { Atenção Básica, } 2012\end{array}$ & $\begin{array}{l}\text { Atualizar os conceitos na política e introduzir } \\
\text { elementos ligados ao papel desejado da AB na } \\
\text { ordenação das Redes de Atenção; avançar no } \\
\text { reconhecimento de um leque maior de modelagens } \\
\text { de equipes para as diferentes populações e realidades } \\
\text { do Brasil; incluir equipes de Atenção Básica para a } \\
\text { população em situação de rua (Consultórios na Rua); } \\
\text { ampliar o número de municípios que podem ter } \\
\text { NASF; facilitar as condições para que sejam criadas } \\
\text { Unidades Básicas de Saúde (UBS) Fluviais e eSF para as } \\
\text { Populações Ribeirinhas. }\end{array}$ \\
\hline $\begin{array}{l}\text { Caderno de Atenção } \\
\text { Básica, no 39, Núcleo de } \\
\text { Apoio à Saúde da Família } \\
\text {-V. 1: Ferramentas para a } \\
\text { gestão e para o trabalho } \\
\text { cotidiano }\end{array}$ & $\begin{array}{c}\text { Ministério da Saúde/ } \\
\text { Secretaria de Atenção à } \\
\text { Saúde/ Departamento de } \\
\text { Atenção Básica, } 2014\end{array}$ & $\begin{array}{l}\text { O documento é elaborado a partir de um conjunto de } \\
\text { oficinas e da colaboração de diversas áreas técnicas do } \\
\text { Ministério da Saúde, no esforço de trazer o acúmulo } \\
\text { de trabalhadores e gestores para lidar com os desafios } \\
\text { vivenciados pelos NASF. Desta forma, sua construção } \\
\text { visa apoiar e ofertar ferramentas para a implantação e } \\
\text { a qualificação do processo de trabalho dessas equipes, } \\
\text { em consonância com as diretrizes da política nacional. }\end{array}$ \\
\hline
\end{tabular}




\section{As diretrizes do NASF como guia do processo de trabalho}

Esta categoria incorpora dados referentes às orientações para a operacionalização do NASF. De modo geral, os documentos analisados ressaltam que o trabalho do NASF é orientado pelas diretrizes da AB, em que as equipes do NASF devem apoiar as eSF na produção de um cuidado continuado e longitudinal, próximo da população e na perspectiva da integralidade (Brasil, 2010a; 2012; 2014).

Nesta proposta, os documentos ressaltam a integralidade como a principal diretriz a ser praticada pelos NASF, compreendida nas suas três dimensões: (1) a prática, que consiste na abordagem integral do indivíduo; (2) a organizacional, que integra as ações de promoção, prevenção, reabilitação e cura, de forma a garantir o acesso às redes de atenção, conforme as necessidades de sua população; e (3) a política, que está relacionada às respostas governamentais a certos problemas de saúde e necessidades de grupos específicos (Brasil, 2010a).

No que se refere à dimensão prática da integralidade, verifica-se que esta se propõe a realização de um cuidado focado na pessoa e nos aspectos cotidianos da sua vida, minimizando as práticas centradas apenas nas doenças. Nesta proposta, há uma valorização dos contextos familiares, sociais e culturais em que cada pessoa se encontra inserida. Além disto, há um reconhecimento do usuário como um sujeito e não como um objeto (Mattos, 2005). Portanto, os documentos destacam que os profissionais da equipe do NASF tenham uma visão abrangente das necessidades dos sujeitos acompanhados e dos contextos sociais em que estão inseridos, ou seja, onde constroem suas histórias de vida.

No tocante aos sentidos pertinentes à organização dos serviços, bem como às respostas governamentais, verifica-se que a integralidade visa: proporcionar maior satisfação das necessidades de saúde da população, por meio da organização do trabalho em saúde; disponibilizar o acesso às técnicas de diagnóstico e tratamento quando necessários, a fim de promover a resolutividade do cuidado; e dar respostas a problemas de saúde, por meio da implantação de políticas especificamente programadas (Mattos, 2005).

Logo, o processo de trabalho do NASF deve garantir o acesso da população a cuidados de saúde, incluindo tanto ações de caráter assistencial e curativo, como atividades de promoção e prevenção, de modo individual e coletivo. Ademais, o NASF deve conhecer o seu território e a oferta de serviços existentes na rede, bem como reconhecer os limites da prática em um serviço de $\mathrm{AB}$, integrando-se aos demais níveis de complexidade do Sistema Único de Saúde (SUS), a fim de satisfazer o conjunto de cuidado demandado por uma pessoa ou por um grupo populacional (Oliveira, \& Quevedo, 2016).

Ressalta-se que, apesar da polissemia da palavra integralidade, pode-se verificar que para o alcance dos diferentes sentidos atribuídos ao termo é preciso colocar em destaque a necessidade de saúde do usuário. Tanto na dimensão prática quanto na dimensão organizacional e política do termo, fica claro que a amplitude da integralidade exige que as equipes do NASF, juntamente com as eSF, reconheçam a grande variedade de necessidades relacionadas à saúde e disponibilize os recursos para abordá-las.

Ainda sobre a integralidade, os documentos analisados apontam para a necessidade de estar atento ao risco de se reproduzir a fragmentação do cuidado, por meio da implantação do NASF (Brasil, 2010a). Ao se incorporar diferentes abordagens disciplinares com o intuito de favorecer a qualidade dos atendimentos, corre-se o risco de cada categoria profissional assumir aquilo que corresponde ao seu núcleo saber, deixando de promover um cuidado integral ao usuário. Esta postura também pode gerar a não responsabilização dos profissionais de saúde, tanto em termos individuais como coletivos, além da focalização de aspectos individuais dos usuários (Oliveira, \& Quevedo, 2016).

O território também é destacado, nos documentos analisados, como um conceito que embasa o trabalho do NASF (Brasil, 2010a; 2014). Por sua vez, o território em saúde não se limita apenas há uma área geográfica. Ele também é compreendido como um espaço que congrega pessoas, instituições, redes, caracterizando-se como um cenário onde se dá a vida comunitária. Assim, trabalhar no território significa conhecer a suas características e seus recursos, a fim de utilizar todos os seus componentes, saberes e forças, para o enfrentamento dos problemas de saúde apresentados (Gazignato, \& Silva, 2014).

Dessa maneira, as equipes do NASF, juntamente com as eSF, devem conhecer a realidade do território sob sua responsabilidade, identificando as suas fragilidades e possibilidades, a fim de propor soluções coletivas condizentes com as suas necessidades de saúde. Além disto, ao conhecer o seu território, 
os documentos apontam também que, as equipes devem identificar os diversos segmentos sociais que o habitam, visando à construção de um trabalho articulado em torno de um tema ou situação em comum, na perspectiva intersetorial (Brasil, 2010a; 2014).

Corrobora-se com Mendes e Donato (2003), ao ressaltarem que a apropriação do território por parte das equipes de $\mathrm{AB}$ (como, eSF e NASF) possibilita a construção de vínculos de corresponsabilização com a comunidade. Nesse sentido, as equipes da $\mathrm{AB}$ assumem a parcela de responsabilidade que lhes cabe, tanto pelas pessoas que habitam o território como pelos espaços em que estes se relacionam.

As práticas de Educação Permanente em Saúde (EPS) e educação popular também aparecem nos documentos analisados como preceitos do trabalho do NASF (Brasil, 2010a; 2014). No que se refere à EPS, sua proposta traz a discussão sobre a relevância e a viabilidade de disseminar a prática pedagógica por todo o sistema de saúde que, segundo Merhy (2005), consistiria em produzirautointerrogação de si mesmo no agir produtor do cuidado. Em outras palavras, a proposta se refere em colocar-se ético-politicamente em discussão, no plano individual e coletivo, problematizando o cotidiano de trabalho. Além disso, a Política Nacional de Educação Permanente (Brasil, 2009) aborda a descentralização do processo de ensino para a configuração locorregional. Desta forma, a EPS deve acontecer no cotidiano dos serviços de saúde, sendo realizada a partir dos problemas enfrentados na realidade, levando em consideração os conhecimentos e as experiências que as pessoas já têm.

Neste contexto, a EPS, desenvolvida pelo NASF, deve promover espaços que fomentem a qualificação profissional, tanto das eSF quanto da sua própria equipe, por meio da problematização do processo de trabalho e das necessidades de formação e de desenvolvimento dos trabalhadores, pautadas pelas demandas de saúde do território. Logo, o NASF, como uma equipe de apoio, deve estimular, propiciar e compor com a eSF espaços críticos-reflexivos e participativos, a fim de promover mudanças no trabalho com a produção de novas ações de saúde norteadas pela prática interdisciplinar e pelo cuidado integral (Miccas, \& Batista, 2014).

Ressalta-se ainda, que a EPS, desenvolvida pelas equipes NASF, não deve se efetivar apenas nos momentos destinados na agenda dos profissionais para esse fim. A EPS deve ser transversal ao cuidado em saúde, favorecendo o desenvolvimento de ações criativas, inovadoras e resolutivas para o enfrentamento dos determinantes e condicionantes sociais do processo saúde-doença dos territórios de responsabilidade das eSF e dos NASF. Assim, considera-se que todos os espaços de interação, diálogo e reflexão sobre a prática em saúde podem ser utilizados para a EPS (reunião de equipe, consulta e visita domiciliar compartilhada, reunião com o conselho gestor, reuniões intersetoriais e de articulação da rede, dentre outros) (Pagani, \& Nascimento, 2016).

Já a educação popular em saúde pressupõe a construção do conhecimento por meio da troca de experiências e conhecimentos, da história de vida, do contexto social e da cultura de cada pessoa. Produzir saberes nesta perspectiva, requer a compreensão de que o conhecimento se produz a partir da instauração do diálogo entre as diferentes concepções de mundo. Além disto, esse dialogar deve ser regido pelo respeito ao outro, considerando que teoria e práticas são distintas, porém inseparáveis e complementares (Freire, 2002).

Espera-se, por meio da educação popular em saúde, empreender uma relação de troca de conhecimentos entre o saber popular e o científico, com enriquecimento recíproco. Reitera-se que tal proposta se mostra cada vez mais necessária, visto que, os modelos tradicionais de saúde reproduziram um distanciamento entre o conhecimento técnico e o popular, criando medidas sanitárias desarticuladas do contexto sociocultural, com poucos impactos na situação de saúde dos coletivos (Gomes, \& Merhy, 2011).

Neste contexto, coaduna-se com Pagani (2016), ao ressaltar que o trabalho do NASF é ampliado e qualificado quando inserido na dinâmica local, conhecendo o contexto cultural do sujeito e da comunidade sobre sua responsabilidade. Assim, para o alcance da diretriz da educação popular em saúde, primeiramente, as equipes do NASF terão que reconhecer e valorizar o saber do outro. Em seguida, é de fundamental importância à criação de espaços que permitam a interação cultural e as negociações entre os diferentes atores envolvidos com a produção de cuidado, para a construção de um novo saber e para a criação de estratégias voltadas a resolução dos problemas de saúde do território.

Ademais, para viabilização da prática de educação popular em saúde, sugere-se com base no Caderno de Educação Popular em Saúde (Brasil, 
2007), o desenvolvimento de algumas ações por parte das equipes dos NASF: (1) participar dos diferentes espaços presente no território (movimentos sociais, atividades culturais, atividades comunitárias, conselho local de saúde, dentre outros), a fim de criar vínculos com a comunidade, compreender seus hábitos, seus costumes, seus conflitos, seu modo de viver e de compreender a vida; (2) conhecer o processo histórico do território e sua relação com o fazer saúde, uma vez que este conhecimento pode ser utilizado como um dos recursos para a melhoria da qualidade de vida da comunidade; (3) identificar as lideranças populares do território para atuarem em parceira nos diferentes projetos de saúde; e (4) valorizar as singularidades e a diversidade das pessoas que fazem parte desta população nas ações de saúde, tanto do caráter individual quanto coletivo.

Os documentos analisados, também ressaltam a humanização como uma prática que orienta o trabalho do NASF (Brasil, 2010a; 2014). De acordo com o Ministério da Saúde (Brasil, 2010b), a Política Nacional de Humanização (PNH) tem como finalidade interferir nos processos de trabalho no campo da saúde, por meio da inclusão de usuários, trabalhadores e gestores na gestão dos serviços. Busca-se a criação de estratégias coletivas, destinadas à promoção de mudanças nos modelos de atenção e de gestão em curso, sempre que tais modelos estiverem na contramão do que preconiza o SUS.

Dito isto, o trabalho do NASF, guiado pelos princípios da PNH, deve se materializar por meio da criação de formas de trabalho que não se submetam à lógica dos modos de funcionamento focados na fragmentação dos sujeitos e do trabalho, mas ao contrário, em "formas de trabalhar que superem as dissociações entre os que pensam e os que fazem, entre os que planejam e os que executam, entre os que geram e os que cuidam". Parte-se da compreensão do trabalho como uma atividade situada em um tempo e espaço coletivo de produção de saberes, de trocas de conhecimentos, de negociação e de gestão (Santos Filho, Barros, \& Gomes, 2009, p. 604).

Outras diretrizes também foram englobadas nos documentos analisados (Brasil, 2010a; 2014), a saber: a interdisciplinaridade, destacada como uma atitude de permeabilidade, a ser tomada pelos profissionais do NASF, frente aos diferentes conhecimentos que podem auxiliar o processo de trabalho e a efetividade do cuidado; a participação social, que visa o fortale- cimento dos espaços comunitários com foco na gestão participativa e no protagonismo dos usuários; e a promoção à saúde realizada tanto ao nível da clínica quanto na condução de atividades coletivas.

Especificamente, para a efetivação da diretriz da promoção à saúde, os documentos apontam para necessidade da organização do trabalho do NASF estar vinculado à garantia de direitos de cidadania e à produção de autonomia de sujeitos e coletividades. Desta forma, pode-se dizer que o trabalho do NASF, organizado na perspectiva da promoção à saúde, deve favorecer o processo de criação coletiva de condições favoráveis à melhoria da qualidade de vida da comunidade como um todo.

Vale ressaltar que, o conceito de promoção à saúde permanece em constante debate teórico no campo da saúde. De modo geral, observa-se que as práticas de promoção à saúde ainda são confundidas, ou compreendidas como sinônimo das práticas de prevenção de doenças, que se relacionam a ocorrência de agravos, tendo como propósito antecipar determinadas ações para evitar ou reduzir o risco do adoecimento (Iglesias, \& Dalbello-Araujo, 2011). Dito isto, para a real efetivação e fomento do princípio da promoção à saúde por meio das equipes NASF, destaca-se a necessidade da incorporação de práticas de educação em saúde, que abordem a Política Nacional de Promoção da Saúde, no nível da AB.

\section{Metodologia e ferramentas de trabalho}

Os dados dessa categoria referem-se ao modo indicado pelos documentos de funcionamento do NASF e dos instrumentos que devem ser utilizados para a operacionalização do trabalho dessas equipes. De acordo com os documentos analisados, o trabalho do NASF é orientado pelo referencial teórico-metodológico do apoio matricial. Em síntese, isso significa que o trabalho acontece a partir da integração entre as eSF e os profissionais dos Núcleos, sendo que essa interação deve se dar a partir das necessidades e dificuldades do território, identificadas pelas eSF (Brasil, 2010a; 2012; 2014).

Dito de outra forma, o matriciamento pressupõe que as equipes de referência, neste caso constituídas pelas eSF, e equipes de apoio matricial mantenham uma relação horizontal entre si, de forma a gerar novos saberes para ambas as equipes e, consequentemente, novas possibilidades de atuação (Campos, \& Domitti, 2007). 
Nesta proposta, cabe ressaltar que todos os saberes são valorizados. Aposta-se na troca de conhecimentos entre as equipes e na ideia de que nenhum especialista, isoladamente, pode assegurar uma abordagem integral à saúde. Para tanto, pode-se afirmar que o apoio matricial se configura como uma "metodologia de trabalho potente na estruturação da rede de saúde por meio da transformação das relações entre os profissionais e, consequentemente, destes com os outros atores sociais, incluindo os usuários" (Iglesias, \& Avellar, 2014, p. 3793).

Reitera-se ainda que, os documentos analisados, enfatizam que o apoio matricial é uma ferramenta que apresenta duas dimensões de suporte: clínico-assistencial e técnico-pedagógica. A dimensão clínico-assistencial é a que busca produzir ação clínica direta com os usuários; a ação técnico-pedagógica, por sua vez, visa produzir ação de formação com e para as equipes envolvidas. Tais dimensões podem e devem se misturar em diversos momentos, como por exemplo, no atendimento compartilhado, na interconsulta, nas práticas gupais, nas ações no território, dentre outras (Brasil, 2010a; 2014).

Os documentos também demostram que, para o desenvolvimento do processo de trabalho do NASF, algumas ferramentas tecnológicas precisam ser adotadas por todas as categorias profissionais que o compõe. Dentre estas, cita-se a Pactuação do Apoio, caracterizada como uma ferramenta de cogestão, e a Clínica Ampliada, o Projeto Terapêutico Singular (PTS) e o Projeto de Saúde no Território (PST), como ferramentas de apoio à atenção (Brasil, 2010a; 2014).

A Pactuação do Apoio configura-se como uma ferramenta que se mantém em constante construção, sendo operada em duas linhas de trabalho. A primeira linha de trabalho visa a avaliação conjunta da situação inicial do território entre os gestores, eSF e o Conselho de Saúde. Antes de definir quais as categorias profissionais irão compor o NASF, é importante que o gestor coordene um processo de discussão e negociação com as eSF e com a participação social, para definir quais profissionais serão contratados. Já a segunda linha envolve a pactuação do desenvolvimento do processo de trabalho e das metas entre os gestores, a equipe do NASF, a eSF e a participação social. Desta forma, essa linha está relacionada ao delineamento dos objetivos a serem alcançados; a identificação dos problemas prioritários a serem abordados; a elaboração dos critérios de encaminhamento ou comparti- lhamento de casos; a criação de mecanismo de avaliação do trabalho da equipe e dos apoiadores; além de formas de explicitação e gerenciamento resolutivo de conflitos (Brasil, 2010a).

No que se refere às ferramentas de apoio à atenção, entende-se a Clínica Ampliada como um cuidado que busca evitar uma abordagem que privilegie somente alguma matriz de conhecimento disciplinar. Além disto, este tipo de clínica assume um compromisso com o usuário, para além da doença, por meio do desenvolvimento da escuta qualificada e do reconhecimento do saber e dos interesses das pessoas, caracterizando-se como uma prática menos prescritiva e mais negociada (Campos, Figueiredo, Pereira Júnior, \& Castro, 2014). Assim, para a incorporação e efetivação desta ferramenta no cotidiano do trabalho do NASF, seus profissionais terão que trabalhar e fomentar, entre as eSF, a compreensão ampliada do processo saúde-doença, promovendo, consequentemente, a ampliação do objeto de trabalho neste nível de complexidade.

Verifica-se que, neste processo de ampliação da clínica, o PTS, também destacado nos documentos analisados como uma ferramenta de trabalho do NASF, pode se configurar como um aliado para o alcance do cuidado integral, uma vez que se defini como: uma estratégia de cuidado que congrega um conjunto de propostas de condutas terapêuticas articuladas para uma pessoa, família ou coletivo, que faz uso tanto dos recursos da equipe, quanto do território, da família e do próprio sujeito. Sua construção ocorre por meio da discussão coletiva de uma equipe interdisciplinar e o usuário sendo, geralmente, aplicado nas situações eleitas como mais complexas (Oliveira, 2007). Cabe destacar que tal discussão "leva em conta as necessidades, as expectativas, as crenças e o contexto social da pessoa ou do coletivo para o qual está dirigido" (Brasil, 2013, p. 55). Sendo assim, o PTS incorpora o olhar da singularidade sobre a situação vivenciada, fomentando a corresponsabilidade de todos os atores envolvidos (profissionais de saúde, usuários e familiares) com o problema de saúde apresentado, por meio de interação democrática e horizontal (Pinto, et al., 2011).

Já o PST se caracteriza como uma ferramenta de trabalho que visa desenvolver ações efetivas de produção de cuidado no território, articulando os serviços de saúde com os demais serviços e políticas sociais, de forma a investir na qualidade de vida e na 
autonomia das comunidades. Seu foco está na promoção à saúde, na participação social e na intersetorialidade (Brasil, 2010a).

De acordo com a análise dos documentos, a construção do PST deve ser iniciada pela identificação de uma área e/ou população vulnerável ou em risco. Após esta etapa, segue-se com: (a) justificativa da priorização da área e/ou população vulnerável; (b) compreensão do processo histórico e social do território; (c) definição dos objetivos das equipes de saúde com relação à área e/ou população; (d) estabelecimento das ações para alcançar os objetivos; (e) identificação de outros atores sociais e/ou instituições importantes para a condução do projeto (Brasil, 2010a).

Em suma, para implantação do PST, as equipes dos NASF e as eSF devem criar espaços coletivos de discussão, visando promover a análise das prioridades e das necessidades de saúde do território, bem como, a identificação dos seus determinantes sociais, a fim elaborar coletivamente estratégias para a melhoria da qualidade de vida e redução das vulnerabilidades no território de adstringência. Reitera-se que "é no espaço coletivo onde a comunidade, suas lideranças e membros de outras políticas e/ou serviços públicos, presentes no território, poderão se apropriar, reformular, estabelecer responsabilidades, pactuar e avaliar o projeto de saúde para a comunidade" (Figueiredo, 2012, p. 65).

É importante ressaltar que essa fase inicial de construção do PST é de responsabilidade das equipes de saúde da $A B$ (eSF com apoio do NASF), mas que o processo não se encerra por aí. Conforme já colocado anteriormente, a proposta do PST busca ampliar a discussão e fomentar a participação social, envolvendo outros setores e atores da sociedade para a mudança da situação identificada em cada território (Verdi, Freitas, \& Souza, 2012).

\section{A organização do trabalho}

Esta categoria apresenta os pressupostos centrais para a sistematização do trabalho do NASF, de acordo com os documentos analisados. Seus dados foram organizados em subcategorias, a saber: (1) planejamento das ações; (2) organização da agenda de trabalho; e (3) acesso ao NASF.

\section{Planejamento das ações}

Os dados referentes à definição e programação das ações a serem desenvolvidas pelos NASF foram agrupados nesta subcategoria. De acordo com os resultados encontrados, nos documentos analisados, o planejamento das ações do NASF deve ser definido e articulado entre sua equipe, eSF e gestores, visando a construção compartilhada de diretrizes clínicas e sanitárias e de critérios para acionar o apoio. Ressalta-se que, tanto na construção destas diretrizes quanto na elaboração dos critérios para a atividade de apoio, deve imperar a flexibilidade e a horizontalidade das relações e responsabilidades. Logo, as ações planejadas devem ser realizadas de forma dinâmica e dialética, sempre sujeitas a reavaliação e reprogramação (Brasil, 2014).

Os documentos apontam também que, o foco do planejamento das ações das equipes NASF deve ser o fortalecimento da $\mathrm{AB}$ e as necessidades das eSF e do território, tendo por base os principais problemas e desafios para o avanço no cuidado integral e resolutivo à saúde da população (Brasil, 2014).

Cabe destacar ainda que, em relação a essa subcategoria "planejamento", não foi identificado na análise dos documentos a inclusão do usuário neste processo. Corrobora-se com Matus (2000) que, para a efetividade do planejamento frente às necessidades do território, faz-se necessário que este ocorra de forma democrática e dialógica, com a presença de todos os atores envolvidos com a ação a ser desenvolvida. $\mathrm{O}$ autor ressalta que, esta forma de planejamento favorece a comunicação, a consciência coletiva e a corresponsabilização.

Organização da agenda de trabalho

Nesta subcategoria foram agrupados os dados referentes às ações que podem ser contempladas na agenda dos profissionais dos NASF. De acordo com os resultados encontrados, nos documentos analisados, o gerenciamento das agendas dos profissionais dos NASF é, em primeira instância, responsabilidade deles. Entretanto, esta deve ser construída de forma compartilhada com as eSF e com o gestor da Unidade Básica de Saúde (UBS), favorecendo o comprometimento de todos os envolvidos com as ações a serem desenvolvidas (Brasil, 2014).

Os documentos apontam ainda para a necessidade de se contemplar nas agendas, as amplas possibilidades de ações que podem ser realizadas no trabalho integrado com as eSF. Dentre estas, destacam-se tanto as ações de caráter técnico-pedagógico (reunião de matriciamento; reunião de equipe NASF; discus- 
sões e construção de PTS; estudo de casos e ações de educação permanente) quando as de caráter clínico-assistencial (atendimento individual compartilhado e específico; atendimento domiciliar compartilhado e específico; atividade coletiva compartilhada e específica). Além disto, também deve ser reservado um espaço na agenda para a elaboração de materiais de apoio e protocolos (Brasil, 2010a; 2014).

Verifica-se que as ações propostas para compor a agenda dos profissionais do NASF, buscam transpor um processo de trabalho que se operacionaliza por meio da fragmentação do cuidado, ao contrário, busca-se o compartilhamento da organização da agenda com os demais profissionais da AB. Corrobora-se com Medeiros et al. (2010) que, de modo geral, o trabalho em saúde não é desenvolvido em conjunto, mas, sim, isoladamente, fato que prejudica uma prática que deveria ser alicerçada na perspectiva da interdisciplinaridade e da integralidade.

Entretanto, cabe ressaltar que a flexibilidade das ações para a composição da agenda dos NASF, destacadas nos documentos analisados, com vistas a promover um trabalho compartilhado com as eSF, não é encontrada nas políticas que norteiam a atuação das eSF. Existe uma grande heterogeneidade do ponto de vista da organização do trabalho entre os profissionais do NASF e da eSF, que dificulta a elaboração de uma agenda conjunta e interfere diretamente na qualidade da relação estabelecida entre essas equipes, a saber: as prioridades das ações; o tempo destinado para a realização das atividades; a produtividade e as metas exigidas, demandas distintas de atendimento populacional, dentre outras situações (Barros, Gonçalves, Kalther, \& Lancman, 2015; Bonaldi, \& Ribeiro, 2014).

\section{O acesso ao NASF}

Esta subcategoria incorpora dados referentes ao acesso tanto das eSF quanto da população as ações desenvolvidas pelas equipes NASF. De acordo com os documentos analisados, o NASF não se constitui como um serviço de porta de entrada para o sistema de saúde, mas sim uma equipe de apoio às eSF. Isto é, os profissionais das equipes NASF não devem exercer o papel de intermediador inicial entre o usuário e o serviço. Nesta proposta, o acesso da população as ações relativas ao núcleo de saber de cada categoria profissional do NASF deve ocorrer, preferencialmente, a partir das discussões dos casos nos encontros de matriciamento. Logo, as intervenções diretas do NASF frente a usuários e famílias, de caráter individual, podem ser realizadas em situações de extrema necessidade, eleitas por meio das discussões, pactuações e negociações a priori entre os profissionais responsáveis pelo caso (Brasil, 2010a; 2014).

Acredita-se que, a orientação dada para que o NASF não se caracterize como um serviço de porta de entrada, seja colocada em função da pressão que existe por parte da população e até mesmo das eSF e dos gestores, para que os profissionais do NASF absorvam a imensa demanda para atendimento individual, tornando-o um ambulatório de especialidades. Sabe-se que historicamente os serviços de saúde vêm sendo organizados de modo a favorecer a prática clínica individual em detrimento das ações coletivas interdisciplinares e intersetoriais. Entretanto, cabe ressaltar que, não é somente o fato do NASF não ser um serviço de porta de entrada que irá determinar a realização de um trabalho integrado e condizente com a proposta da $\mathrm{AB}$, mas sim, o modo como é operacionalizado suas diretrizes e as demais orientações, colocadas nas políticas, direcionadas ao processo de trabalho do NASF.

Destaca-se ainda que, conforme demostrado no estudo realizado por Silva et al. (2011), com profissionais que compõem as equipes NASF, não existe um consenso entre esses sobre o fato do usuário não ter acesso direto aos profissionais do NASF, ou seja, alguns profissionais têm a ideia que deveria existir uma possibilidade de agendamento direto com os membros das equipes NASF. Infere-se que essa resistência, seja fruto de uma dificuldade de desenvolver um trabalho conjunto, uma vez que as normativas que discorrem sobre a forma de acessar o NASF, produzem mudanças na lógica de encaminhamento presente nos serviços de saúde, incitando um trabalho compartilhado e corresponsável. Logo, esse modo de acionar os profissionais do NASF busca romper com as relações verticais dos mecanismos de referências e contrarreferências, em que a comunicação entre os profissionais de saúde ocorre somente por meio de relatos escritos, em uma lógica de transferência de responsabilidade e passagem de informes (Bonaldi, \& Ribeiro, 2014; Oliveira, \& Quevedo, 2016).

Os dados, obtidos por meio da análise dos documentos, ressaltam a importância de que sejam evitadas: a criação de pastas de encaminhamentos de casos para as equipes NASF; a disponibilização de agendas 
na recepção das UBS para casos de demanda espontânea ou encaminhados por outros serviços sem pactuação prévia com as eSF; e outras ações que não se utilizem da lógica da discussão centrada na pessoa, da pactuação e da construção conjunta entre as equipes da AB (Brasil, 2014).

Os documentos analisados apontam ainda que, o acesso direto aos profissionais do NASF por meio da população, pode acontecer a partir de outras modalidades de cuidado, desde que estas sejam coerentes com os princípios da $\mathrm{AB}$, articuladas com as eSF e com os demais setores interessados. Dentre estas modalidades, destaca-se: intervenções no território, como por exemplo, no desenvolvimento de PST; apoio a grupos e ações coletivas; trabalhos educativos e de inclusão social; enfrentamento de situações de violência e ruptura social e ações de caráter intersetorial (Brasil, 2014).

No que se refere ao acesso das eSF ao NASF, os dados revelam que, além deste ocorrer por meio de encontros de matriciamento e das outras formas de interação entre ambas as equipes, devem ser definidos critérios de risco e meios para o acionamento do NASF em situações urgentes e/ou imprevistas (Brasil, 2014).

De modo geral, os documentos analisados relatam que existem diversos caminhos para o acesso ao NASF. Entretanto, estes caminhos devem ser construídos conjuntamente entre as equipes do NASF e eSF, bem como com a gestão da UBS e municipal, favorecendo o comprometimento de todos os envolvidos com as pactuações realizadas. Observa-se que neste processo, a comunicação e a interação entre os profissionais do Núcleo e da eSF são recursos fundamentais para pactuar formas de acesso ao NASF, condizentes com as demandas da população e das próprias eSF.

\section{Considerações finais}

Por meio dos resultados obtidos neste estudo, foi possível verificar que o trabalho do NASF dispõe de um leque de possibilidade de ações a serem desenvolvidas por "meio de alianças" entre os profissionais da $\mathrm{AB}$, gestores, comunidade e demais equipamentos sociais existentes no território. Para tanto, faz-se necessário à criação espaços de comunicação rotineiros, de caráter interno e externo, para a discussão de casos, definição de objetivos em comuns a serem trabalhados, metas a serem alcançadas, critérios de prioridades, avaliação, resolução de problemas, den- tre outros. Além disto, tais espaços devem tencionar a reorganização dos serviços a fim de superar a lógica fragmentada do cuidado em saúde, potencializando o trabalho em rede e integral.

Todavia, pondera-se a existência de alguns desafios e entraves a serem enfrentados para a consolidação da proposta de trabalho do NASF. Acredita-se que instituir um diálogo em rede se constitui um dos maiores desafios para as equipes NASF, uma vez que, a efetivação desta proposta requer o desenvolvimento de um trabalho compartilhado e de cogestão das ações, que deve ser operado de forma horizontal entre os diferentes serviços que compõem a rede. Em outras palavras, para a consolidação do trabalho em rede é preciso considerar tanto os vínculos construídos entre os diferentes serviços existentes no território, como a própria capacidade de articulação da Rede de Atenção à Saúde (RAS) com os diferentes pontos de atenção existentes.

Outro entrave para a efetivação da proposta do NASF, diz respeito à heterogeneidade existente entre o seu trabalho e o das eSF. Desta forma, verifica-se a necessidade de uma revisão das normativas direcionadas as eSF, visando favorecer a realização do trabalho compartilhado entre essas equipes (eSF e NASF). Por fim, como último desafio destacado neste estudo, ressalta-se a importância da inserção do usuário nos espaços de planejamentos das ações a serem desenvolvidas pelas equipes NASF, visando tanto garantir a oferta do cuidado de forma coerente com as necessidades do território, como o fomento do protagonismo, da autonomia e da corresponsabilidade desses com processo saúde doença.

Em síntese, por meio dos resultados obtidos neste estudo, pode-se afirmar que o trabalho do NASF deve ser construído coletivamente, por meio das pactuações realizadas a partir da construção de um olhar apurado sobre as demandas de saúde do território adstrito. Neste cenário, os processos de interação e pactuação se constituem em elementos-chave para a condução do trabalho do NASF condizente com as suas diretrizes. Entretanto, ressalta-se a necessidade de ficar atento para o modo como estão sendo operados estes elementos no cotidiano do trabalho. Eles estão enfatizando o caráter inovador do NASF? Possibilitam a atuação integrada, com o desenvolvimento de ações compartilhadas e coletivas centradas no cuidado integral? 
A despeito dessas questões, verifica-se que para a efetivação da proposta do NASF, torna-se necessário a consolidação de um modelo de atenção pautado pela integralidade e orientado pelo trabalho colaborativo, cujos objetivos visem à superação da lógica biomédica, que ainda se faz presente tanto na formação em saúde quanto nas práticas do cuidado. Nesse processo de reorientação do modelo de atenção, destaca-se a importância das equipes da $\mathrm{AB}$ possuírem clareza da proposta do trabalho do NASF, proporcionando, assim, o fortalecimento do trabalho integrado e interdisciplinar.

Vale ressaltar ainda que, atualmente, vive-se um paradoxo no contexto da $\mathrm{AB}$. De um lado estão às práticas profissionais, que não conseguem responder de forma adequada às novas necessidades dos cuidados de saúde (integralidade, visão ampliada do processo saúde-doença, formação de vínculos, protagonismo dos sujeitos, realização de trabalhos coletivos e integrados). Por outro lado, existe uma lógica mercantilista que impõe uma produção quantitativa e uma rigidez nos processos de trabalho, por meio de normatização excessiva, da baixa capacidade de desenvolver o princípio da cogestão e da grande dependência dos serviços secundários.

Por sua vez, entende-se que o trabalho na $A B$ não comporta a racionalidade da lógica mercantil, uma vez que o cuidado neste nível de complexidade é pautado no direito de cidadania e no fomento da participação social. Sugere-se para o enfrentamento desse cenário, a análise minuciosa das particularidades do trabalho em saúde, neste nível de complexidade, a fim de compreender a sua dimensão no compromisso com a defesa da vida e, consequentemente, com a concretização de um sistema de saúde universal e de qualidade.

Ressalta-se também, que os documentos analisados nesse estudo, englobam questões normativas e organizacionais que orientam o funcionamento do NASF. Entretanto, a operacionalização do trabalho das equipes NASF irá ocorrer no cotidiano dos serviços, sendo esse um espaço com diversos atravessamentos: a interface entre o saber e o poder, a intersubjetividade, os aspectos presentes no encontro dos trabalhadores entre si e destes com o usuário, dentre outros aspectos. Desta forma, para que os documentos normativos tenham relevância e aplicabilidade no trabalho em saúde, sua elaboração tem que ocorrer por meio de processos de negociação e reformulação, a partir da experiência vivenciada nos serviços entre os atores sociais envolvidos no processo de cuidado em saúde.

Por fim, espera-se com esta pesquisa gerar reflexões de modo a contribuir para a condução de processos de trabalho guiados pelos princípios e diretrizes do NASF. Certamente, a construção de uma prática conforme preconiza o NASF é um desafio, porém o alcance da sua proposta é de extrema importância para o fortalecimento do SUS.

\section{Referências}

Anjos, K. F., Meira, S. S., Ferraz, C. E. O., Vilela, A. B. A., Boery, R. N. S. O., \& Sena, E. L. S. S. (2013). Perspectivas e desafios do núcleo de apoio à saúde da família quanto às práticas em saúde. Saúde em Debate, 37(99), 672-680. https://doi.org/10.1590/S0103-11042013000400015.

Bardin, L. (2009). Análise de conteúdo. Lisboa: 70.

Barros, J. O., Gonçalves, R. M. A., Kaltner, R. P., \& Lancman, S. (2015). Estratégia do apoio matricial: A experiência de duas equipes do núcleo de apoio à saúde da família (NASF) da cidade de São Paulo, Brasil. Ciência \& Saúde Coletiva, 20(9), 2847-2856. https://doi.org/10.1590/1413-81232015209.12232014

Bonaldi, A. P., \& Ribeiro, M. D. (2014). Núcleo de apoio à saúde da família: As ações de promoção da saúde no cenário da estratégia saúde da família. Revista de Atenção Primária da Saúde, 17(2), 195-203.

Brasil. Ministério da Saúde. (2007). Caderno de educação popular e saúde. Brasília, DF: o autor.

Brasil. Ministério da Saúde. (2009). A política nacional de educação permanente em saúde para o SUS. Brasília, DF: o autor.

Brasil. Ministério da Saúde. (2010a). Diretrizes do NASF: Núcleo de apoio a saúde da família. Brasília: o autor.

Brasil. Ministério da Saúde. (2010b). HumanizaSUS: Documento base para gestores e trabalhadores do SUS (4a ed.). Brasília, DF: o autor.

Brasil. Ministério da Saúde. (2012). Política nacional de atenção básica. Brasília, DF: o autor. 
Brasil. Ministério da Saúde. (2013). Saúde mental. Brasília, DF: o autor.

Brasil. Ministério da Saúde. (2014). Núcleo de apoio à saúde da família: Ferramentas para a gestão e para o trabalho cotidiano. Brasília, DF: o autor.

Campos, G. W. S., \& Domitti, A. C. (2007). Apoio matricial e equipe de referência: Uma metodologia para gestão do trabalho interdisciplinar em saúde. Cadernos de Saúde Pública, 23(2), 399-407. https://doi.org/10.1590/S0102$311 X 2007000200016$

Campos, G. W. S., Figueiredo, M. D., Pereira Júnior. N., \& Castro, C. P. (2014). A aplicação da metodologia Paideia no apoio institucional, no apoio matricial e na clínica ampliada. Interface: Comunicação, Saúde, Educação, 18(1), 983-995. https://doi.org/10.1590/1807-57622013.0324

Figueiredo, E. N. (2012). A estratégia saúde da família na atenção básica do SUS. São Paulo, SP: Universidade Federal de São Paulo. Recuperado de http://www.unasus.unifesp.br/biblioteca_virtual/pab/1/unidades_conteudos/ unidade06/unidade06.pdf

Freire, P. (2002). Pedagogia da autonomia: Saberes necessários à prática educativa (25a ed.). São Paulo, SP: Paz e Terra.

Gazignato, E. C. S., \& Silva, C. R. C. (2014). Saúde mental na atenção básica: O trabalho em rede e o matriciamento em saúde mental na estratégia de saúde da família. Saúde Debate, 38(101), 296-304. https://doi.org/10.5935/01031104.20140027

Gonçalves, R. M. A., Lancman, S., Sznelwar, L. I., Cordone, N. G., \& Barros, J. O. (2015). Estudo do trabalho em núcleos de apoio à saúde da família (NASF), São Paulo, Brasil. Revista Brasileira de Saúde Ocupacional, 40(131), 59-74. https://doi.org/10.1590/0303-7657000078013

Gomes, L. B., \& Merhy, E. E. (2011). Compreendendo a educação popular em saúde: Um estudo na literatura brasileira. Caderno de Saúde Pública, 27(1), 7-18. https://doi.org/10.1590/S0102-311X2011000100002

Iglesias, A. \& Avellar, L. Z. (2014). Apoio matricial: Um estudo bibliográfico. Ciência \& Saúde Coletiva, 19(9), 3791-3798. https://doi.org/10.1590/1413-81232014199.00322013

Iglesias, A., \& Dalbello-Araujo, M. (2011). As concepções de promoção da saúde e suas implicações. Cadernos Saúde Coletiva, 19(3), 291-298.

Mattos, R. (2005). Os sentidos da integralidade: Algumas reflexões acerca de valores que merecem ser defendidos. In: R. Pinheiro, \& R. Mattos (Orgs.), Os sentidos da integralidade na atenção e no cuidado em saúde (4a ed.). Rio de Janeiro, RJ: Centro de Estudos e Pesquisa em Saúde Coletiva.

Matuda, C. G., Pinto, N. R. S., Martins, C. L., \& Frazão, P. (2015). Colaboração interprofissional na estratégia saúde da família: Implicações para a produção do cuidado e a gestão do trabalho. Ciência \& Saúde Coletiva, 20(1), 2511-2521. https://doi.org/1413-81232015208.11652014.

Matus, C. (2000). O líder sem estado-maior. São Paulo, SP: Fundação do Desenvolvimento Administrativo.

Medeiros, C. R. G., Junqueira, A. G. W., Schwinge, G., Carreno, I., Jungles, L. A. P., \& Saldanha, O. M. F. L. (2010). A rotatividade de enfermeiros e médicos: Um impasse na implementação da estratégia de saúde da família. Ciências \& Saúde Coletiva, 15(supl. 1), 1521-1531. https://doi.org/10.1590/S1413-81232010000700064

Mendes, R., \& Donato, A. F. (2003). Território: Espaço social de construção de identidades e de políticas. Sanare: Revista de Políticas Públicas, 4(1), 39-42.

Merhy, E. E. (2005). O desafio que a educação permanente tem em si: A pedagogia da implicação. Interface: Comunicação, Saúde, Educação, 9(16), 172-174. https://doi.org/10.1590/S1414-32832005000100015

Miccas, F. L., \& Batista, S. H. S. S. (2014). Educação permanente em saúde: Metassíntese. Revista de Saúde Pública, 48(1), 170-185. https://doi.org/10.1590/S0034-8910.2014048004498

Oliveira, G. N. (2007). O projeto terapêutico como contribuição para a mudança das práticas de saúde. Dissertação de Mestrado, Universidade Estadual de Campinas, Campinas, SP.

Oliveira, F. R., \& Quevedo, M. P. (2016). Núcleo de apoio a saúde da família (NASF) e a construção das redes de atenção à saúde. In: M. L. M. Santos, V. L. Kodjaoglanian, \& F. P. Ferrari, (Org.), O NASF em cena: Tecnologias e ferramentas de trabalho no cotidiano das equipes (pp. 113-144). Porto Alegre, RS: Rede Unida.

Oliveira, M. M. (2007). Como fazer pesquisas qualitativas. Petrópolis, RJ: Vozes. 
Pagani, R. (2016). A educação popular em saúde e o fazer/aprender: interagir/agir do núcleo de apoio à saúde da família. In: M. L. M. Santos, V. L. Kodjaoglanian, \& F. P. Ferrari (Org.), O NASF em cena: Tecnologias e ferramentas de trabalho no cotidiano das equipes (pp. 85-112). Porto Alegre, RS: Rede Unida.

Pagani, R., \& Nascimento, D. D. G. (2016). Equipe do núcleo de apoio à saúde da família: Criar e recriar as possibilidades do seu fazer no cotidiano da saúde. In: M. L. M. Santos, V. L. Kodjaoglanian, \& F. P. Ferrari (Org.), O NASF em cena: Tecnologias e ferramentas de trabalho no cotidiano das equipes (pp. 51-84). Porto Alegre, RS: Rede Unida.

Paim, J. S. (2003). Modelos de atenção e vigilância em saúde. In: M. Z. Rouquayrol, \& N. Almeida Filho (Orgs.), Epidemiologia e saúde (pp. 567- 586, 6a ed.). Rio de Janeiro, RJ: Medsi.

Pinto, D. M., Jorge, M. S. B., Pinto, A. G. A., Vasconcelos, M. G. F., Cavalcante, C. M., \& Flores A. Z. T. (2011). Projeto terapêutico singular na produção do cuidado integral: Uma construção coletiva. Texto \& Contexto: Enfermagem, 20(3), 493-302. https://doi.org/10.1590/S0104-07072011000300010

Portaria no 154, de 24 de janeiro de 2008. Cria os núcleos de apoio à saúde da família - NASF. Diário Oficial da União, 25 jan. 2008.

Santos Filho, S. B. S., Barros, M. E. B., \& Gomes, R. S. (2009). A política nacional de humanização como política que se faz no processo de trabalho em saúde. Interface: Comunicação, Saúde, Educação, 13(1), 603-613. https://doi. org/10.1590/S1414-32832009000500012

Silva, A.T.C., Aguiar, M.E.A., Winck, K., Rodrigues, K.G.W., Sato, M.E., Grisi, S.J.F.E. et al. (2011). Núcleos de Apoio à Saúde da Família: Desafios e potencialidades na visão dos profissionais da Atenção Primária do Município de São Paulo, Brasil. Cadernos de Saúde Pública, 28(11), 2076-2084. https://doi.org/10.1590/S0102311X2012001100007

Verdi, M. I. M., Freitas, T. G., \& Souza, T. T. (2012). Projeto de saúde no território. Florianópolis, SC: Universidade Federal de Santa Catarina.

\section{Meyrielle Belotti}

Doutora em Psicologia na Universidade Federal do Espírito Santo (UFES). Mestre em Psicologia Institucional pela UFES. Possui graduação em Terapia Ocupacional pela Faculdade Integrada São Pedro. Professora Adjunto de Departamento de Terapia Ocupacional da UFES. Tem experiência no campo da saúde coletiva atuando principalmente nos seguintes temas: saúde mental, atenção básica, apoio matricial, intersetorial e rede.

E-mail: meyri.belotti@gmail.com

https://orcid.org/0000-0003-3901-4656

\section{Alexandra Iglesias}

Graduada em psicologia pela Universidade Federal do Espírito Santo (UFES). Mestre em saúde coletiva pela mesma Universidade. Doutora pelo Programa de Pós-Graduação em Psicologia da UFES. Professora Adjunta da UFES/ Departamento de Psicologia. Tem experiência nas áreas de saúde coletiva, saúde mental e psicologia da saúde, atuando principalmente com os seguintes temas: promoção à saúde, apoio matricial, saúde mental e atenção básica.

E-mail: leiglesias@gmail.com

https://orcid.org/0000-0001-7188-9650

\section{Luziane Zacché Avellar}

Possui graduação em Psicologia pela Universidade Federal do Espírito Santo (UFES), mestrado em Psicologia Social pela Pontifícia Universidade Católica (PUC) de São Paulo, doutorado em Psicologia Clínica pela PUC de São Paulo. Atualmente é professora Titular da UFES. Tem experiência na área de Psicologia, atuando principalmente nos seguintes temas: saúde, saúde mental e saúde mental infanto juvenil.

E-mail: luzianeavellar@yahoo.com.br

https://orcid.org/0000-0003-3125-2174 
Psicologia: Ciência e Profissão 2019 v. 39, e185025, 1-14.

Recebido 06/09/2017

Aceito 01/03/2019

Received 09/06/2017

Approved 03/01/2019

Recibido 06/09/2017

Aceptado 01/03/2019

Como citar: Belotti, M., Iglesias, A., \& Avellar, L. Z. (2019). Análise documental sobre as normativas do trabalho no núcleo ampliado de saúde da família. Psicologia: Ciência e Profissão, 39, 1-14. https://doi.org/10.1590/1982-3703003185025

How to cite: Belotti, M., Iglesias, A., \& Avellar, L. Z. (2019). Documentary analysis on labor standards at the family health expanded support center. Psicologia: Ciência e Profissão, 39, 1-14. https://doi.org/10.1590/1982-3703003185025

Cómo citar: Belotti, M., Iglesias, A., \& Avellar, L. Z. (2019). Análisis documental sobre las normativas del trabajo en el núcleo ampliado de salud de la familia. Psicologia: Ciência e Profissão, 39, 1-14. https://doi.org/10.1590/1982-3703003185025 